\title{
Mitoxantrone Hydrochloride
}

National Cancer Institute

\section{Source}

National Cancer Institute. Mitoxantrone Hydrochloride. NCI Thesaurus. Code C665.

The hydrochloride salt of an anthracenedione antibiotic with antineoplastic activity.

Mitoxantrone intercalates into and crosslinks DNA, thereby disrupting DNA and RNA

replication. This agent also binds to topoisomerase II, resulting in DNA strand breaks and

inhibition of DNA repair. Mitoxantrone is less cardiotoxic compared to doxorubicin. 